\title{
Management of pulmonary embolism
}

\author{
H H Gray, S Firoozan
}

Pulmonary embolism may be subclinical, representing an apparently incidental finding at necropsy, ${ }^{1}$ or may result in varying degrees of haemodynamic disturbance. In the United States around 650000 symptom producing cases of pulmonary embolism have been estimated to occur each year, contributing to the death of around 200000 patients $^{2}$ and representing the sole cause of death in $50000 .^{3}$ In the United Kingdom an estimated 20000 patients die each year in hospital as a consequence of pulmonary embolism and $\mathbf{4 0 0 0 0}$ have non-fatal episodes. ${ }^{4}$ Others have reported pulmonary embolism as contributing to 15$20 \%$ of deaths in an acute general hospital. ${ }^{5}$ Individual reports of the mortality and incidence of pulmonary embolism disappointingly have shown little change over the years despite the advent of new treatments, ${ }^{67}$ in part perhaps because of the increase in the elderly population. An overview of more than 70 randomised trials with 16000 patients does, however, suggest that the perioperative use of subcutaneous heparin can prevent about half of pulmonary embolisms and about two thirds of deep venous thromboses, with a substantial reduction in fatal episodes. ${ }^{8}$ The diagnosis of pulmonary embolism is usually made on clinical grounds but accurate confirmation depends on the results of pulmonary angiography, radionuclide lung scanning, or necropsy. Such confirmatory evidence is often unavailable, which explains why figures cited for the incidence and mortality of pulmonary embolism must be regarded at best as approximations. Because of its ubiquitous nature, the responsibility for managing patients with pulmonary embolism falls to clinicians of many specialties; perhaps not surprisingly, the management of such patients varies considerably, and is at times less than ideal. ${ }^{9}$

\section{Aetiology}

Virchow described the classical clinical triad that predisposes to venous thromboembolism - namely, local trauma to a vessel wall, hypercoagulability, and blood stasis. ${ }^{10}$ Most patients with pulmonary embolism have clinical conditions known to be associated with these predisposing factors, including major trauma, recent surgery, obesity and immobility, increasing age, malignant disease, use of the oral contraceptive pill, late pregnancy and the first few weeks after delivery, and less common condi- tions, such as the hyperviscosity and nephrotic syndromes.

A few patients with pulmonary embolism have an underlying primary clotting abnormality that rencers their blood hypercoagulabfe, such as defective fibrinolysis, ${ }^{112}$ antithrombin III deficiency, the presence of lupus anticoagulant, ${ }^{13-16}$ and congenital deficiencies in protein $\mathrm{C}$ and its cofactor protein $\mathrm{S}$. These coagulation abnormalities are uncommon when taken in the context of pulmonary embolism as a whole and routine investigation for all patients may not be cost effective. In younger patients, however, those with a family history of thromboembolism, and those with recurrent embolic episodes with no obvious cause, screening is justified. Air; amniotic fluid; and deposits of tumour, fat, and foreign materials such as fragments of central venous catheters may all embolise to the lungs; but by comparison with embolisation of thrombus these causes are rare and will not be considered further.

\section{Pathophysiology}

The clinical effects of pulmonary thromboembolism depend on the extent of pulmonary vascular obstruction; whether vasoactive and bronchoconstricting humoral agents, such as serotonin and thromboxane $A_{2},{ }^{17}$ are released from activated platelets; whether any pre-existing cardiopulmonary disease is present; and the age and general health of the patient. These factors help to explain the widely varying clinical presentation of patients with pulmonary embolism and reinforce the need for a flexible approach towards management. Thus a young and otherwise fit individual may tolerate and recover spontaneously from a $25 \%$ occlusion of the pulmonary circulation, whereas an elderly or infirm individual may suffer severe circulatory collapse.

Of these factors, the most important and the most amenable to therapeutic intervention is the extent of pulmonary vascular obstruction. It has been estimated that right ventricular afterload increases when more than a quarter of the pulmonary circulation is obstructed. Initially right ventricular pressure rises, leading to dilatation and tricuspid regurgitation. As the right ventricle begins to fail pressure falls. Hence the finding of only a slight rise in pulmonary artery pressure may indicate either a modest degree of obstruction of the pulmon- 
ary artery or severe obstruction with failure of the right ventricle. In the largest studies observations made during the course of pulmonary embolism suggest that an otherwise normal right ventricle is incapable of acutely increasing the pressure of the pulmonary arterial much above $50-60 \mathrm{~mm} \mathrm{Hg}$ in response to sudden major obstruction of the pulmonary circulation. By contrast, in chronic vascular obstruction, such as occurs in thromboembolic or primary pulmonary hypertension, right ventricular pressure may rise gradually to suprasystemic levels.

The haemodynamic effect of pulmonary embolism are not confined to the right heart. Obstruction of pulmonary arterial flow causes a reduction in left heart filling (preload) ${ }^{18}$ and dilatation of a failing right ventricle results in displacement of the interventricular septum into the left ventricular cavity, ${ }^{19}$ further impairing left ventricular filling and reducing end diastolic volume. ${ }^{18}$ These effects help explain why the dyspnoea of patients with acute severe obstruction of the pulmonary circulation is eased by manoeuvres that increase systemic venous return and consequently left ventricular preload such as lying flat, Trendelenberg titting, and infusing colloid intravenously. This is in contrast to the dyspnoea of patients with left ventricular failure, which is eased by sitting upright and by interventions aimed at reducing left ventricular preload, such as diuretic treatment.

\section{Clinical presentations}

Many small emboli are likely to go undetected clinically. ${ }^{20}$ Because pulmonary embolism so frequently occurs under conditions of associated illness, its effects may go unnoticed by both patient and clinician. A high level of clinical suspicion is required if such oversight is to be avoided, expecially in patients who fall within the high risk categories discussed earlier.

Various classifications of the severity of pulmonary embolism have been used, such as the Walsh score $^{21}$ in the United States and the Miller score $^{22}$ in Europe. These represented attempts to quantify angiographically the degree of pulmonary obstruction and were aimed, in part, at measuring the change in obstruction brought about by treatment. They served a useful purpose, but the clinical manifestations of pulmonary embolism depend on more than the extent of pulmonary arterial obstruction. For this reason a clinical classification has been suggested ${ }^{23}$ that aims to describe the haemodynamic consequences of the embolus in an individual patient and thus assist in the choice of treatment.

Acute minor pulmonary embolism is a consequence of obstruction of small distal pulmonary arteries, often resulting in infarction. When symptoms occur they include mild tachypnoea, pleuritic chest pain, and haemoptysis. There is usually no haemodynamic disturbance. Physical examination often shows no abnormality but may reveal tachycardia, a pleural rub, and mild pyrexia.

Acute major pulmonary embolism results from substantial obstruction of the proximal pulmonary arteries and usually causes severe dyspnoea, dull central chest pain, tachycardia, gallop rhythm, raised venous pressure, and tachypnoed. When the degree of pulmonary arterial obstruction is sudden and severe, syncope or death may ensue.

Chronic thromboembolic pulmonary hypertension frequently presents with a gradual onset of dyspnoea, with or without a prior history of venous thrombosis or pulmonary emboli, and is due to chronically increasing pulmonary arterial obstruction resulting from factors such as unresolved acute massive embolism, recurrent smaller emboli, and thrombosis in situ. Chest discomfort may occur during exertion. The findings produced by examination depend on the severity of pulmonary hypertension, and are representative of the degree of right ventricular pressure overload.

These consequences of pulmonary embolism determine the clinical differences in presentation (table I). Unfortunately clinical symptoms and signs discriminate poorly between the presence and the absence of pulmonary embolism as determined by pulmonary angiography, ${ }^{24}$ and if clinical suspicion exists further investigations are indicated.

\section{Investigations}

Electrocardiography shows characteristic abnormalities only in massive pulmonary embolism as a result of the acute increase in right ventrieutar aftertoad. These include the classical S1, Q3, T3 pattern; right bundle branch block; p pulmonale; and right axis deviation. One or a combination of these occurred in only $26 \%$ of patients in the large Urokinase Pulmonary Embolism Trial. ${ }^{25}$ Other changes include sinus tachycardia, atrial

Table 1 Clinical differences between the various presentations of pulmonary embolism

\begin{tabular}{llll}
\hline & Acute minor & Acute major & Chronic \\
\hline Dyspnoea & Mild & Severe & Chronic, progressive \\
Chest pain & Pleuritic & Acute, dull, central & Exertional, dull, central \\
Tachycardia & Mild & Usually severe & Variable \\
Blood pressure & Normal & Low & Normal until late \\
Cyanosis & No & Common & Common \\
Oedema & No & Not acutely & Common \\
Jugular venous pressure & Normal & Raised & Raised \\
Heart sounds & Normal & S3 & S3, S4, P2+ \\
Chest radiograph & Often normal & Usually abnormal & Abnormal \\
Electrocardiogram & Usually normal & Usually abnormal & Abnormal \\
Systolic pulmonary artery pressure & Normal & $30-50$ & $>70$ \\
(mm Hg) & & & \\
\hline
\end{tabular}


Table 2 Differential diagnosis of pulmonary embolism

Myocardial infarction

Pneumonia

Asthma

Pneumothorax

Acute pulmonary oedema

Aortic dissection

Pleurisy

Fractured rib

Musculoskeletal pain

Right or left heart disease

Cor pulmonale

fibrillation, and $T$ wave inversion in the anteroseptal leads.

Chest radiographic changes are also frequently non-specific. Dilatation of a major proximal pulmonary artery and areas of pulmonary oligaemia may suggest major arterial obstruction. Wedge shaped opacities in the peripheral lung fields due to pulmonary infarction, with or without a small pleural effusion, may occur with minor pulmonary embolism. In chronic thromboembolic pulmonary hypertension the cardiothoracic ratio may be increased with features suggesting dilatation of the right ventricle. Patchy oligaemia and dilatation of the main pulmonary arteries may be present. Overall, if pulmonary embolism is suspected the chest radiograph is more helpful in excluding alternative diagnoses, such as lobar pneumonia (table 2).

Measurement of arterial blood gas tensions is relatively unhelpful diagnostically but may be useful in assessing the response to treatment. Patients with pulmonary embolism are not always hypoxaemic, though this becomes increasingly likely with increasing degrees of pulmonary arterial obstruction. Hypoxaemia may obviously be due to several other causes. $A$ blood test that could be easily performed to diagnose pulmonary embolism has long been sought, but there is so far no equivalent of the creatinine phosphokinase MB test used in the diagnosis of myocardial infarction. In conditions where thrombus is formed plasmin mediated proteolysis of fibrin releases D dimeric fragments (D dimer), which can be quantified. D dimer has been found in $89 \%$ of patients with pulmonary embolism confirmed by ventilation $(\bar{V}) /$ perfusion (Q) isotope lung scanning, but also in $56 \%$ of those with negative scans, rendering it relatively nonspecific. A D dimer concentration below 500 $\mathrm{ng} / \mathrm{ml}$ has been suggested as excluding pulmonary embolism, ${ }^{26}$ though a negative isotope lung scan is a better criterion.

Pulmonary function tests may give abnormal results, but are again non-specific, though they may be of some benefit in assessing functional recovery after pulmonary embolism. ${ }^{27}$ Physiological dead space (VD) and the ratio of $V D$ to tidal volume ( $\nabla T)$ increase. In one investigation $\mathrm{VD} / \mathrm{VT}$ was found to be over $40 \%$ in 16 patients with pulmonary embolism but $40 \%$ or Tess in 29 where pulmonary embolism was excluded. ${ }^{28}$

Isotope radionuclide ventilation-perfusion $(\dot{V} / \dot{Q})$ lung scanning is perhaps the most widely available investigation currently used in suspected pulmonary embolism, but it has its limitations.
Often only a perfusion scan is obtained, which if normal essentially rules out pulmonary embolism and can therefore be helpful for management decisions. Abnormal perfusion, however, occurs in conditions other than pulmonary embolism, such as chronic lung disease and pneumonia. When an assessment of ventilation is made simultaneously, evidence of $\dot{V} /$ Q mismatch greatly increases the likelihood of pulmonary embolism. Radionuclide scans are usually reported as representing a low, moderate, of high likelihood of pulmonary embolism. Indeterminate scans may arise when the chest radiograph shows chronic pulmonary disease or is abnormal in the region of the perfusion defect. Eighty six per cent of patients with high probability scans have been shown to have angiographic evidence of pulmonary emboli, whereas only $32 \%$ of those with scans of lower probability had angiographic evidence. ${ }^{29}$ In the large prospective investigation of pulmonary embolism diagnosis (PIOPED) trial $^{3031}$ only $41 \%$ of patients with angiographically proved pulmonary embolism had high probability isotope scans, $59 \%$ having scans of lower probability. Patients with high, intermediate, and low probability scans had positive predictive values for angiographically proved pulmonary embolism of $87 \%, 32 \%$, and $16 \%$. Near normal scans were associated with pulmonary embolism in $9 \%$ of cases. When suggesting pulmonary embotism, radionuclide scans tend to underestimate the associated angiographic severity and haemodynamic disturbance of pulmonary embolism.

Other radionuclide techniques include labelling platelets ${ }^{33}$ or monoclonal antifibrin antibodies ${ }^{34}$ with indium, but these are probably more relevant to the diagnosis of deep venous thrombosis than pulmonary embolism.

Pulmonary angiography has historically been regarded as the diagnostic gold standard, but it is a highly invasive procedure and is not available in many hospitals. ${ }^{35}$ It would be foolish to suggest that all patients suspected of having pulmonary embolism should have such an investigation merely because isotope lung scanning is imperfect. A more practical approach is required. Patients with a normal isotope perfusion scan are extremely unlikely to have pulmonary embolism, and in those cases where the index of clinical suspicion is high and the isotope lung scan suggests that pulmonary embolism is probable the diagnosis can be considered confirmed. These two groups of patients require angiography only exceptionally. Where the index of clinical suspicion is moderate or high and the isotope scan is equivocal, however, pulmonary angiography should be considered. ${ }^{36}$ If the angiogram is negative anticoagulation can be avoided and if it is positive the extent and severity of pulmonary arterial obstruction can be determined and consideration given to the various treatments available. Pulmonary angiography has acquired a reputation for being a dangerous procedure, probably because some deaths were associated with the procedure in the early days of its use. With advances in technique and 
newer contrast media, however, the risk of the procedure is small. ${ }^{37}$ Of the 755 patients undergoing angiography in the PIOPED trial the procedure contributed to death in two $(0.3 \%)$, both of whom had the most severe disease. ${ }^{30}$ In experienced hands pulmonary angiography is also safe in those with chronic thromboembolic pulmonary hypertension. ${ }^{38}$ Relative contraindications include pregnancy, known right atrial thrombus, and hypersensitivity to contrast media. Abnormalities sought on angiograms include filling defects, suggesting thrombus, and areas of pulmonary oligaemia. Multiple images of the lung fields should be obtained, magnified views being used if necessary. Cut films and digital imaging techniques are superior to cineangiography. Digital subtraction angiography may be adequate for showing proximal pulmonary arterial occlusions, but is less good at detecting more peripheral obstruction. $^{39} 40$

Echocardiography is widely available but usually contributes little diagnostically. In breathless adults the quality of the images may not be good. Right heart dilatation may be noted and if tricuspid regurgitation is found by means of Doppler techniques right ventricular pressure can be estimated. Thrombus in the right atrium or inferior vena cava may be seen, especially if transoesophageal echocardiography is used. ${ }^{41}$

Investigations of the deep venous system As the primary source of pulmonary emboli is from thrombus arising in the deep veins of the pelvis and legs and the risk of pulmonary embolism at least partly depends on the location and extent of deep vein thrombosis, ${ }^{9}$ several investigations aimed at the deep venous system have been developed. Contrast venography has been the historic gold standard against which the newer techniques of impedance plethysmography, radiofibrinogen leg scanning, and Duplex (Doppler flow assessment combined with ultrasound scanning) have been assessed. Their accuracy in detecting deep vein thrombosis depends to a large extent on the experience of the operator. As pulmonary embolism implies that deep vein thrombosis is present and both conditions dictate anticoagulation, little may be gained from investigations specific to the deep venous system. Thrombolytic agents achieve more rapid resolution of deep vein thrombosis than heparin, but have not been shown to be better than standard anticoagulants in lessening the risk of further episodes or in reducing postphlebiric symptoms. ${ }^{42}$ Where the clinical suspicion of pulmonary embolism is not high and radionuclide lung scans are equivocal, positive results of investigations suggesting deep vein thrombosis, especially proximal to the calf, dictate anticoagulation. ${ }^{4}$

Computed tomography ${ }^{44}$ and magnetic resonance imaging ${ }^{45}{ }^{46}$ have been used in the diagnosis of pulmonary embolism but their place remains uncertain.

\section{Treatments}

Apart from supportive treatment such as analgesia and oxygen, the three treatment options for pulmonary embolism are anticoagulation, thrombolytic treatment, and pulmonary embolectomy.

\section{ANTICOAGULATION}

Heparin accelerates the action of antithrombin III and prevents further fibrin deposition, allowing the body's fibrinolytic system to lyse existing clot. If the partial thromboplastin time is prolonged before heparin is started the possibility of lupus anticoagulant should be considered, and in these circumstances the concentration of heparin itself can be assayed. There is a tendency to use too little anticoagulant $t^{47}$ and those with deep vein thrombosis and pulmonary embolism may require more heparin to achieve adequate anticoagulation than those without active thrombosis. ${ }^{48}$

Only one randomised trial has compared heparin with placebo in the management of pulmonary embolism, which was halted after 35 patients had been enrolled because of a significantly higher mortality in the placebo group. ${ }^{49}$ The investigators concluded that heparin did not reduce the immediate mortality of pulmonary embolism, but probably reduced deaths due to further emboli. Despite this, many still use heparin as a single drug for patients with circulatory collapse after massive pulmonary embolism, even though at best this prevents further emboli only while the patient's fibrinolytic system acts to dissolve the obstructing thrombus.

\section{THROMBOLYSIS}

When thrombolytic agents became available a National Institutes of Health consensus conference concluded that they represented "a significant advance in the management of the more severe forms of pulmonary embolism, ${ }^{50}$ though even now such agents are used infrequently. Several controlled trials have compared thrombolytic agents with heparin their unequivocal conclusion being that pulmonary emboliclear more rapidly with the former. ${ }^{2251-56}$ Although mortality tended to be lower in the thrombolysis group, the difference nevertheless failed to achieve statistical significance. This may have been due to the fact that patients who survived long enough to be entered into the trial represented a group with a better prognosis, because the most severely affected pacients would have died before receiving thrombolytic therapy. ${ }^{57}$ As the randomised groups then became those with a better prognosis, the number of patients required to detect a difference was probably greater than the trials enrolled.$^{52}$ Despite the lack of statistical proof of prognostic benefit, achieving more rapid resolution of pulmonary emboli seems desirable as prolonged haemodynamic disturbance can only harm the patient and further emboli, should they occur, would have an adverse haemodynamic effect. A second potential advantage of thrombolysis is that potentially dangerous iliofemoral venous thrombi may be lysed, ${ }^{58}$ though there is always the concern (not proved to have any substance) that this may result in detachment of a clot from the vessel wall, resulting in further pulmonary embolism. 
There is no evidence that thrombolytic treatment results in better long term restoration of pulmonary blood flow than heparin.

\section{PULMONARY EMBOLECTOMY}

The place of pulmonary embolectomy remains controversial. ${ }^{37}$ The prognostic benefit of surgical removal of emboli has never been tested in a clinical trial and is unlikely ever to be so, given the ethical impossibility of randomising patients to surgery when the less invasive treatment of thrombolysis has been shown to be effective. Thus surgery can only be justified if the patient is thought unlikely to survive without operation. Some patients are so severely compromised haemodynamically that the clinician may feel that they will not survive the hour or two required to derive initial benefit from thrombolysis. Thrombolytic drugs may also be contraindicated in some patients, and others may deteriorate despite their administration. For the few patients with massive pulmonary embolism who fall into these groups pulmonary embolectomy may be lifesaving. ${ }^{59}$

Using the surgical technique of venous inflow oclusion, which does not require cardiopulmonary bypass, Clarke and Abrams have shown that embolectomy can be performed successfully in any hospital with surgical facilities, with an in hospital mortality of around $20 \% .{ }^{60}$ In those centres with the necessary equipment the procedure is usually undertaken on dardiopulmonary bypas, with similar results. ${ }^{61}$ Embolectomy can also be undertaken transvenously ${ }^{62}$ using a suction device, though this technique has not been widely used. If

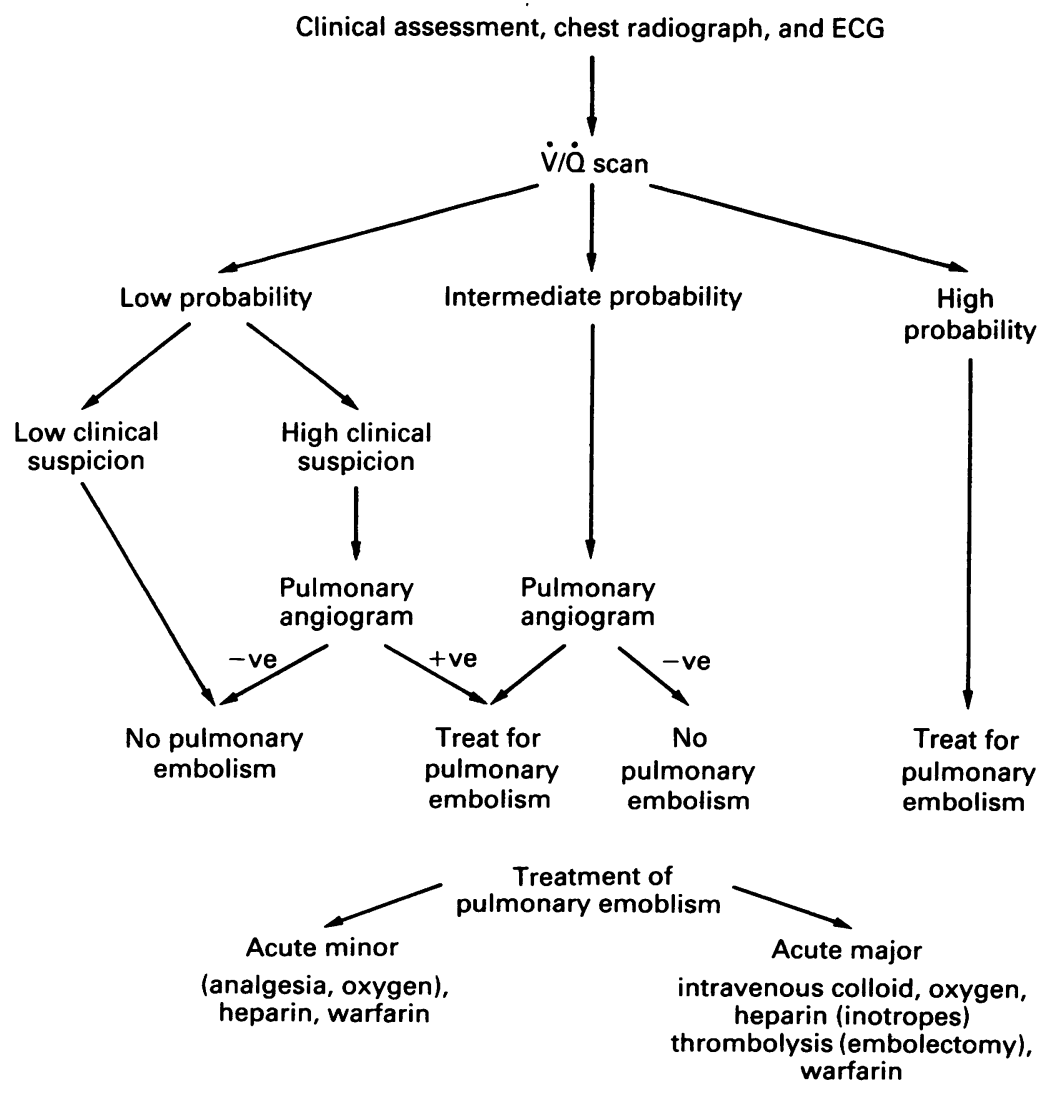

Management of pulmonary embolism. embolectomy is considered, pulmonary angiography must be undertaken, partly to identify the site and extent of pulmonary arterial obstruction, but mainly to ensure that the diagnosis is correct. Patients mistakenly undergoing surgical embolectomy for alternative diagnoses, such as myocardial infarction, have a high mortality.

\section{PROCEDURES ON THE INFERIOR VENA CAVA}

Once a pulmonary embolism has occurred, residual thrombus is almost always present in the deep veins, having been found at necropsy in over $90 \%$ of cases. ${ }^{9}$ Attempts have been made to prevent further emboli by surgical interruption or plication of the inferior vena cava, but nowadays percutaneous insertion of devices such as filters and umbrellas is more common. These techniques have been applied more widely in the United States than Europe and their efficacy has never been established in a randomised clinical trial. It might be difficult to show prognostic benefit because the rate of recurrence of pulmonary embolism with treatment regimens not using these devices is remarkably low (around 5\%) and patients rarely die from further embolism after hospital discharge. ${ }^{63}$ After acute massive pulmonary embolism in 71 patients treated without inferior vena cava intervention recurrent emboli were uncommon ( $6 \%$ ) and no cases of clinical pulmonary hypertension were found in nine years. ${ }^{59}$ In the absence of continuing risk factors progression to chronic thromboembolic pulmonary hypertension is rare. In patients with persistent venous thrombosis following pulmonary embolism thrombolytic treatment has been suggested to be more effective than inferior vena cava interruption in preventing further episodes. ${ }^{64}$ In the acute phase of massive pulmoñary embolism any procedure that reduces venous return is potentially detrimental. For those patients at high risk of further emboli and in whom anticoagulation is contraindicated, or for those who have recurrent emboli despite adequate anticoagulation, an inferior vena cava filter may be beneficial.

Management (figure)

A clear history should be obtained, both of the patient's symptoms and of potential risk factors. With few exceptions, patients suspected of having pulmonary embolism should have chest radiography and electrocardiography, and be referred for a radionuclide $V / Q$ lung scan. Where this is negative and clinical circumstances allow, it may be appropriate to manage the patient at home, but the vast majority of patients should be admitted to hospital. Thrombus in the pulmonary arteries has almost always come from elsewhere in the venous system and the first episode of pulmonary embolism may only be one of many. Until the clinical position is clearer patients should be investigated, monitored, and treated in hospital. As many of the risk factors for pulmonary embolism apply solely to hospitalised patients most individuals are likely to be inpatients already.

All those managing patients who fall into the 
risk categories for pulmonary embolism, which encompasses most hospitalised patients, must be alert to the diagnosis because clinical suspicion is the first step towards appropriate investigation and treatment. Once pulmonary embolism is suspected confirmation should always be sought. An incorrect diagnosis commits the patient to months of anticoagulant treatment and its associated risks, and as the presenting features of so many other conditions may be similar to pulmonary embolism it is vital to make an accurate diagnosis. Given that the patient is not haemodynamically compromised and radionuclide scanning can be obtained, it is reasonable to wait until the result of this is known before anticoagulation is started. The subsequent management depends upon the degree of haemodynamic disturbance.

\section{ACUTE MINOR PULMONARY EMBOLISM}

Patients with no haemodynamic disturbance can be given analgesia and oxygen as initial treatment. Those with evidence of peripheral pulmonary emboli from $\dot{V} / Q$ scanning should have heparin initially as there is evidence that this is superior to oral anticoagulation alone. ${ }^{65}$ The degree of anticoagulation required is another uncertainty but it should probably be sufficient to keep the activated partial thromboplastin time in the range $1.5-2.5$ times control values. Loading with an oral anticoagulant is usually undertaken simultaneously. A reasonable strategy is to give $100 \mathrm{IU} / \mathrm{kg}$ heparin as a loading dose, followed by an infusion of $1000-$ $2000 \mathrm{IU} /$ hour. This conventional policy of anticoagulation has not been shown to alter prognosis but it seems logical to attempt to prevent further clot propagation in the deep veins while the patient's fibrinolytic system gradually removes existing thrombus in the veins and pulmonary circulation. Whether heparinisation for longer than is required to achieve adequate oral anticoagulation has any advantage is unknown. Oral anticoagulation is given to achieve an international normalised ratio (INR) of $2 \cdot 0-3 \cdot 0$ and is usually continued for 3-6 months, though the ideal duration is unknown. ${ }^{66}$ Low molecular weight subcutaneous heparin has a longer half life, less variability in the anticoagulation response to a fixed dose, and a more favourable antithrombotic to haemorrhagic ratio than conventional unfractionated heparin and it has been shown to be as effective as intravenous heparin for the treatment of deep vein thrombosis, allowing patients to be treated at home without the need for regular measurements of clotting indices. ${ }^{67}$ Whether such treatment is appropriate for patients with minor pulmonary embolism is unknown. Some but not all trials have shown that the risk of bleeding complications increases with the degree of anticoagulation, ${ }^{68}$ and the risk of bleeding may be lower with continuous intravenous heparin than with intermittent intravenous bolus doses. ${ }^{69}$ The half life of heparin is around 60-90 minutes and the partial thromboplastin time returns to normal in around two to four hours. The effect of heparin can be reversed more rapidly with protamine sulphate (1 mg/100 IU heparin) if serious bleeding occurs. A mild reduction in platelet count is often seen in patients, which is reversed when treatment ceases. More rarely, thrombocytopenia is associated with thrombosis and may have serious consequences. ${ }^{70} 71$

\section{ACUTE MAJOR PULMONARY EMBOLI}

Patients presenting with haemodynamic disturbances (pronounced dyspnoea, hypotension, raised venous pressure, and hypoxaemia) require different management and resuscitation should be undertaken before investigation. Patients should be placed supine, if necessary in the Trendelenburg position, given oxygen, and moved to an intensive care or high dependency unit where appropriate haemodynamic and respiratory monitoring can be performed. The outcome of acute massive pulmonary embolism must be better in an environment where the clinical progress of patients can be closely observed by medical and nursing staff, and management decisions reassessed at frequent intervals. A central venous line should be inserted to allow the measurement of venous pressure and the administration of intravenous colloid and inotropes. ${ }^{72}$ Where thrombolytic treatment is considered a jugular rather than infraclavicular approach should be used. When centralvenous access is achieved the possibility of inserting a flow directed, balloon tipped thermodilution pulmonary artery catheter should be considered as this permits serial measurments of cardiac output to be made. Pulmonary artery pressure and mixed venous oxygen saturation can also be monitored, which helps to assess the rate of resolution of the pulmonary arterial obstruction in response to treatment. Where central venous monitoring is not available transcutaneous oximetry may provide an assessment of resolution. Where thrombolytic treatment is considered, the insertion of arterial lines should be avoided if possible.

In some cases the diagnosis of pulmonary embolism may be so certain on clinical grounds and the patient in need of such urgent treatment that a decision may be made to start thrombolytic treatment without radionuclide scanning or pulmonary angiography. This should not be undertaken lightly in view of the potential hazards of such potent drugs, especially where the diagnosis is incorrect.

Although some patients with major pulmonary embolism may recover spontaneously, ${ }^{73}$ many die soon after the onset of symptoms. Of those who survive an hour or more, many die in the succeeding hours or days. ${ }^{7475}$ Vigorous treatment may hasten the resolution of a major embotus and should be offered to all suitable patients. They should receive thrombolytic treatment unless contraindicated and pulmonary embolectomy should be considered if this cannot be used or is ineffective. Results from trials of streptokinase have suggested that an intravenous loading dose of 250 000-600000 IU over $20-30$ minutes, with or without $100 \mathrm{mg}$ hydrocortisone, followed by $100000 \mathrm{IU} /$ hour intravenously for up to 72 hours is appropriate, followed by warfarin and if necessary heparin until adequate oral anticoagulation is achieved. 
Whether 1.5 MU streptokinase, as in the treatment of myocardial infarction, has the same effect is unknown. Urokinase has been used in the United States in preference to streptokinase and has been compared with tissue plasminogen activator. Trials have suggested more rapid resolution of pulmonary thrombus with tissue plasminogen activator than urokinase but their effects are similar after 12-24 hoûrs.

\section{CHRONIC THROMBOEMBOLIC PULMONARY HYPERTENSION}

The insidious nature of chronic thromboembolic pulmonary hypertension means that it often presents late, at a stage where there is often little alternative to pulmonary thromboendarterectomy ${ }^{76}$ or lung or heart-lung transplantation. Although vasodilators have been used to treat chronic pulmonary hypertension these might be expected to be relatively ineffective where the primary abnormality is pulmonary arterial occlusion by fixed organised thrombus, though they may help to recruit the remaining pulmonary vessels. Domiciliary oxygen may help symptomatically.

\section{Conclusion}

Despite prophylactic measures pulmonary embolism remains a common problem, presenting to a wide range of clinicians. The management of patients varies considerably, partly because of the absence of data from randomised clinical trials but also through a lack of familarity with the management of such patients. In most areas of medicine increasing the practice of a skill improves performance. A similar improvement in the management and outcome of pulmonary embolism might be achieved if experience were concentrated in the hands of fewer clinicians. Such a strategy would facilitate the performance of clinical trials and help to answer some of the many outstanding questions relating to pulmonary embolism.

1 Goldman L, Sayson R, Robbins S, et al. The value of the autopsy in three medical eras. $N$ Engl J Med 1983, 308:1000-5.

2 Bell WR, Simon TL. Current status of pulmonary thromboembolic disease: pathophysiology, diagnosis, prevention and treatment. Am Heart J 1982;103:239-62.

3 Coon WW, Willis PW. Deep venous thrombosis and pulmonary embolism: prediction, prevention and treatment. Am J Cardiol 1959;4:611-21.

4 Tibbutt DA, Chesterman CN. Pulmonary embolism current therapeutic concepts. Drugs 1976;11:161-92.

5 Morrell MT, Dunnill MS. The post-mortem incidence of pulmonary embolism in a hospital population. $\mathrm{Br} J$ Surg 1968;55:347-52.

6 Goldhabor SZ. Pulmonary embolism death rates. Am Heart J 1988;115:1342-3.

7 Lindblad B, Sternby NH, Bergquist D. Incidence of venous thromboembolism verified by necropsy over 30 years. BMJ 1991;302:709-11.

8 Collins R, Scrimgeour A, Yusuf S, Peto R. Reduction in fatal pulmonary embolism and venous thrombosis by perioperative administration of subcutaneous heparin. Overview of results of randomised trials in general, orthopedic and urologic surgery. N Engl J Med 1988; 318:1162-73.

9 Moser KM. Venous thromboembolism. Am Rev Respir Dis 1990;141:235-49.

10 Virchow R. Gesammelte Abhandlungen zur Wissenschaftlichen Medizin. Frankfurt: Meidinger, 1856:219.

11 Stead NW, Bauer KA, Kinney TR, et al. Venous thrombosis in a family with defective release of vascular plasminogen activator and elevated plasma factor VIII/von Willebrand's factor. Am J Med 1983;74:33-9.
12 Pizzo SV, Fuchs HE, Doman KA, et al. Release of tissue plasminogen activator and its fast-acting inhibitor in defective thrombolysis. Arch Intern Med 1986;146: 188-91.

13 Branch DW, Scott JR, Kochenour NK, Hershgold E. Obstetric complications associated with lupus anticoagulant. N Engl J Med 1985;313:1322-6.

14 Petri M, Rheinschmidt M, Whiting-O'Keefe $Q$, et al. The frequency of lupus anticoagulant in systemic lupus erythematosus. A study of 60 consecutive patients by activated partial thrombolplastin time, Russell viper venom, and anticardiolipin antibody level. Ann Intern Med 1987; 106:524-31.

15 Triplett DA, Brandt JT, Musgrave KA, Orr CA. The relationship between lupus anticoagulants and antibodies to phospholipid. JAMA 1988;259:550-4.

16 Alving BM, Barr CF, Tang DB. Correlation between lupus anticoagulants and anticardiolipin antibodies in patients with prolonged activated partial thromboplastintimes. $\mathrm{Am}$ J Med 1990;88:112-6.

17 Manny J, Hectman HB. Vasoactive humoral factors. In: Goldhaber SZ, ed. Pulmonary embolism and deep venous thrombosis. Philadelphia: Saunders, 1985:283.

18 Belenkie I, Dani R, Smith ER, Tyberg JV. Ventricular interaction during experimental acute pulmonary embolism. Circulation 1988;78:761-8.

19 Jardin F, Dubourg O, Gueret P, et al. Quantitative twodimensional echocardiography in massive pulmonary embolism: emphasis on ventricular interdependence and leftward septal displacement. JACC 1987;10:1201-6.

20 Freiman DG, Suyemoto J, Wessler S. Frequency of pulmonary thromboembolism in man. $N$ Engl J Med 1965; 272:1278-80.

21 Walsh PN, Greenspan RH, Simon M, et al. An angiographic severity index for pulmonary embolism. Circulation 1973; 47 (suppl 2): 101-8.

22 Miller GAH, Sutton GC, Kerr IH, et al. Comparison of streptokinase and heparin in treatment of isolated acute massive pulmonary embolism. $B M J 1971 ; \mathrm{ii}: 681-4$.

23 Miller GAH. Pulmonary embolism. In: Westherall DJ, Ledingham JGG. Warrell DA. Oxford textbook of medicine. Vol 2. Oxford: Oxford University Press, 1987 355-60.

24 Goldhaber SZ. Strategies for diagnosis. In: Goldhaber SZ ed. Pulmonary embolism and deep venous thrombosis. Philadelphia: Saunders, 1985:79.

25 Stein PD, Dalen JE, McIntyre KM, et al. The electrocardiogram in acute pulmonary embolism. Prog Cardiovasc Dis 1975;17:247-57

26 Bounameaux $\mathrm{H}$, Cirafici $\mathrm{P}$, de Moerloose $\mathrm{P}$, et al. Measurement of D-dimer in plasma as diagnostic aid in suspected pulmonary embolism. Lancet 1991;337: 196-200.

27 Prediletto R, Paoletti P, Fornai E, et al. Natural course of pulmonary embolism. Evaluation by perfusion lung scintigraphy, gas exchange and chest roentgenogram. Chest 1990;97:554-61.

28 Burki NK. The dead space to tidal volume ratio in the diagnosis of pulmonary embolism. Am Rev Respir Dis 1986;133:679-85.

29 Hull RD, Hirsh J, Carter CJ, et al. Diagnostic value of ventilation-perfusion lung scanning in patients with suspected pulmonary embolism. Chest 1985;88:819-28.

30 The PIOPED investigators. Value of the ventilation/perfusion scan in acute pulmonary embolism. Results of the prospective investigation of pulmonary embolism diagnosis (PIOPED). JAMA 1990;263:2753-9.

31 Stein PD, Alavi A, Gottschalk A, et al. Usefulness of noninvasive diagnostic tools for diagnosis of acute pulmonary embolism in patients with a normal chest radiograph. $A m$ $J$ Cardiol 1991;67:1117-20.

32 Ryan KL, Fedullo PF, Davis GB, et al. Pedrfusion scan findings understate the severity of angiographic and hemodynamic compromise in chronic thromboembolic pulmonary hypertension. Chest 1988;93:1180-5.

33 Ezekowitz MD, Pope CF, Sostman HD, et al. Indium-111 platelet scintigraphy for the diagnosis of acute venous thrombosis. Circulation 1986;73:668-74.

34 Jung $M$, Kletter $K$, Dudczak $R$, et al. Deep venous thrombosis: Scintigraphic diagnosis with indium-111labelled monoclonal antifibrin antibodies. Radiology 1989;173:469-75.

35 Kohn H, Kohler D. Diagnostic modalities for detection of pulmonary embolism in clinical routine: European survey. Lung 1990; suppl: 833-40.

36 Robinson PJ. Lung scintigraphy-doubt and certainty in the diagnosis of pulmonary embolism. Clin Radiol 1989; 40:557-60.

37 Gray HH, Miller GAH, Paneth M. Pulmonary embolectomy: its place in the management of pulmonary embolism. Lancet 1988; i:1441-5.

38 Nicod $\mathrm{P}$, Peterson $\mathrm{K}$, Levine $\mathrm{M}$, et al. Pulmonary angiography in severe chronic pulmonary hypertension. Ann Intern Med 1987:107:565-8.

39 Pond GD. Pulmonary digital subtraction angiography. Radiol Clin North Am 1985;23:243-60.

40 Mussett D, Rosso J, Petitprez P, et al. Acute pulmonary embolism: Diagnostic value of digital subtraction 
angiography. Radiology 1988;166:455-9

41 Hunter JJ, Johnson KR, Karagianes TG, Dittrich HC. Detection of massive pulmonary embolus-in-transit by transesophageal echocardiography. Chest 1991;100: 1210-4.

42 Goldhaber SZ, Buring JE, Lepnick RJ, Hennekens $\mathrm{CH}$ Pooled analysis of randomised trials of streptokinase and heparin in phlebographically documented acute deep venous thrombosis. Am J Med 1984;76:393-7.

43 Raskob GE, Hull RD. Diagnosis and management of pulmonary thromboembolism. Q J Med 1990;280:787-97.

44 Chintipalli $\mathrm{K}$, Thorsen MK, Olson DL, et al. Computed tomography of pulmonary thromboembolism and infarction. J Comput Assist Tomogr 1988;12:553-9.

45 Szucs RA, Rehr RB, Tatum JL. Pulmonary artery thrombus detection by magnetic resonance imaging. Chest 1989 95:232.

46 Posteraro RH, Sostman HD, Spritzer CE, Herfkens RJ. Cine-gradient-refocused MR imaging of central pulmonary emboli. AJR 1989;152:465-8.

47 Wheeler AP, Jaquiss RDB, Newman JH. Physician practice in the treatment of pulmonary embolism and deep venous thrombosis. Arch Intern Med 1988;148:1321-5.

48 Hirsh J, van Aken WG, Gallus AS, et al. Heparin kinetics in venous thrombosis and pulmonary embolism. Circulation 1976;53:691-5.

49 Barritt DW, Jordan SC. Anticoagulant drugs in the treatment of pulmonary embolism: a controlled trial. Lancet 1960;i:1309-12.

50 Thrombolytic therapy in treatment. Summary of an NIH consensus conference. $B M J 1980 ; \mathrm{i}: 1585-7$.

51 National Cooperative Study. Urokinase pulmonary embolism trial phase $T$ results. $J A M A$ 1970;214:2163-72.

52 National Cooperative Study. Urokinase pulmonary embolism trial. Circulation 1973;48 (suppl 2):1-108.

53 Tibbutt DA, Davies JA, Anderson JA, et al. Comparison by controlled trial of streptokinase and heparin in treatment of life-threatening pulmonary embolism. BMJ 1974; i: 343-7.

54 Ly B, Arnesen $\mathrm{H}$, Eie $\mathrm{H}$, Hol R. A controlled clinical trial of streptokinase and heparin in the treatment of majo pulmonary embolism. Acta Med Scand 1978;203:465-70

55 Verstraete $M$, Miller $\mathrm{GAH}$, Bounameaux $\mathrm{H}$, et al. Intravenous and intrapulmonary recombinant tissue-type plasminogen activator (rt-PA) in the treatment of acute massive pulmonary embolism. Circulation 1988;77 353-60.

56 Goldhaber SZ, Vaughan DE, Markis JE, et al. Acute pulmonary embolism treated with tissue plasminogen activator. Lancet 1986:ii:886-9.

57 Turnier E, Hill JD, Kerth WJ, Gerbode F. Massive pulmonary embolism. Am J Surg 1973;125:611-22.

58 Mavor GE, Dhall DP, Dawson AA, et al. Streptokinase therapy in deep-vein thrombosis. Br J Surg 1973;60:
468-74.

59 Gray HH, Morgan JM, Paneth M, Miller GAH. Pulmonary embolectomy for acute massive pulmonary embolism: an analysis of 71 cases. Br Heart J 1988;60:196-200.

60 Clarke DB, Abrams LD. Pulmonary embolectomy: a 25 year experience. $J$ Thorac Cardiovasc Surg 1986;92:442-5.

61 Del Campo C. Pulmonary embolectomy: a review. Can J Surg 1985;28:111-3.

62 Greenfield LJ, Langham MR. Surgical approaches to thromboembolism. Br J Surg 1984;71:968-70.

63 Hall RJC, Sutton GC, Kerr IH. Long-term prognosis of treated acute massive pulmonary embolism. Br Heart $J$ 1977;39:1128-34.

64 Haissaguerre M, Bonnet J, Douvier JJ, Bricaud H. Comparison of fibrinolytic treatment with interruption of the inferior caval vein in the prevention of pulmonary embolism. Int $J$ Cardiol 1986;12:213-21.

65 Anonymous. Management of venous thromboembolism [editorial]. Lancet 1988;i:275-7.

66 Petitti DB, Strom BL, Melmon KL. Duration of warfarin anticoagulant therapy and the probabilities of recurrent thromboembolism and haemorrhage. Am J Med 1986 81:255-9.

67 Prandoni $P$, Lensing AWA, Buller HR, et al. Comparison of subcutaneous low-molecular weight heparin with intravenous standard heparin in proximal deep-vein thrombosis. Lancet 1992;339:441-5.

68 Landfeld CS, Cook EF, Flatley M, et al. Identification and preliminary validation of predictors of major bleeding in hospitalised patients starting anticoagulant therapy. $\mathrm{Am} \mathrm{J}$ Med 1987;82:703-13.

69 Hyers TM, Hull RD, Weg JG. Antithrombotic therapy for venous thromboembolic disease. Chest 1989;95 (2 suppl): 37-51S.

70 Rao AK, White GC, Sherman L, et al. Low incidence of thrombocytopenia with porcine mucosal heparin. Arch Intern Med 1989;149:1285-8.

71 Rankin JA. heparin-induced thrombosis (white clot syndrome) secondary to prophylactic subcutaneous administration of heparin. Can J Surg 1988;31:33-4.

72 Prewitt RM. Hemodynamic management in pulmonary embolism and acute hypoxemic respiratory failure. Crit Care Med 1990;18:S61-9.

73 Sautter RD, Fletcher FW, Emanuel DA, Lawton BR, Olsen TG. Complete resolution of massive pulmonary thromboembolism. JAMA 1964;189:948-9.

74 Gorham LW. A study of pulmonary embolism. Arch Intern Med 1961;108:418-26.

75 Linder F, Schmitz W, Encke A, Trede M, Storch HH. A study of 605 fatal pulmonary embolisms and two successful embolectomies. Surg Gynaecol Obstet 1967;125:82-6.

76 Moser KM, Auger WR, Fedullo PF. Chronic major-vessel thromboembolic pulmonary hypertension. Circulation 1990;81:1735-43. 